\title{
THE NUMBER OF PATHS AND CYCLES IN A DIGRAPH*
}

\author{
Dorwin Cartwright and Terry C. Gleason \\ UNIVERSITY OF MICHIGAN
}

\begin{abstract}
An algorithm is presented for constructing from the adjacency matrix of a digraph the matrix of its simple $n$-sequences. In this matrix, the $i, j$ entry, $i \neq j$, gives the number of paths of length $n$ from a point $v_{i}$ to a point $v_{i}$; the diagonal entry $i, i$ gives the number of cycles of length $n$ containing $v_{i}$. The method is then generalized to networks - that is, digraphs in which some value is assigned to each line. With this generalized algorithm it is possible, for a variety of value systems, to calculate the values of the paths and cycles of length $n$ in a network and to construct its value matrix of simple $n$-sequences. The procedures for obtaining the two algorithms make use of properties of a line digraph - that is, a derived digraph whose points and lines represent the lines and adjacency of lines of the given digraph.
\end{abstract}

In research on such topics as cognition, learning, verbal behavior, communication, sociometry, and social interaction, empirical structures are often represented by digraphs (directed graphs) in which each point corresponds to an empirical entity and each directed line corresponds to an empirical relationship. A problem frequently encountered in working with digraphs is to find the number of ways one can go from one point to another, using a given number of lines, without passing through any point more than once. Thus, for example, in the context of communication research, one may want to know how many ways a message can go from one person to another through a network in exactly $n$ steps while satisfying the requirement that no person hear the message more than once. Stated in the terminology of digraph theory, the problem is to find the number of paths of length $n$ from one point to another. In solving this problem, it is necessary to deal also with (directed) cycles. We say that a point lies on a cycle of length $n$ if it is possible to leave a point and then return to it in exactly $n$ steps without passing through any other point more than once.

It is well known that if a digraph $D$ contains no cycles, the number of paths of length $n$ from a point $v_{i}$ to a point $v_{i}$ is given by the $i, j$ entry of $A^{n}$, where $A$ is the adjacency matrix of $D$. But if $D$ has any cycles, the number of paths of length $n$ cannot be ascertained directly from $A^{n}$. There have been several attempts to overcome this limitation. Luce and Perry [4] showed

*The research reported here was supported by Grant NSF-G-17771 from the National Science Foundation. We wish to thank Professor Frank Harary for suggesting certain ways of improving an earlier draft of this paper. 
how to find the paths of length 3 in any digraph. Ross and Harary [6] extended the solution to lengths 4,5 , and 6 and presented an algorithm by which any formula for longer paths could eventually be obtained. However, the formula for length 6 is quite formidable and there seems to be little likelihood that a general solution is practicable by their method. Parthasarathy [5] has offered a solution in principle but its usefulness is limited by the great amount of calculation required.

In this paper we again raise the problem and present a method for finding both the number of paths and cycles of any given length through a series of reasonably simple matrix operations. The crux of the solution lies in exploiting the properties of line digraphs as developed by Harary and Norman [1]. We begin with a discussion of line digraphs and their relevance to the problem, then present a matrix method which capitalizes on the properties of line digraphs, and conclude by generalizing the method to networks in which each line has an assigned value. (For a systematic treatment of digraph theory, see Harary, Norman, and Cartwright [2].)

\section{Digraphs and Line Digraphs}

A directed graph (or digraph) is a non-empty set $V$ of points and a prescribed subset of the set of all ordered pairs of the members of $V$. Each of these ordered pairs $\left(v_{i}, v_{i}\right)$ is called a line, which we denote by $v_{i} v_{i}$ or by $x_{i j}$. The first member of the pair is called the first point of the line and the second member is called the second point. For any digraph we require that there are no lines whose first and second points are the same and every pair of points $v_{i}$ and $v_{i}$ has at most one line $v_{i} v_{i}$.

A (point-line) sequence in a digraph is an alternating sequence of points and lines which begins and ends with a point and has the property that each line is preceded by its first point and followed by its second point. If $L$ is a sequence $v_{i}, x_{i j}, v_{j}, x_{i k}, v_{k}, \cdots, v_{m}, x_{m n}, v_{n}$, we may denote $L$ simply by indicating the order of occurrence of its points: $L=v_{i}, v_{i}, v_{k}, \cdots$, $v_{m}, v_{n}=L_{i j k \cdots m n}$. A sequence is a path if all of its points are distinct. A cycle consists of a path from a point $u$ to a point $v$ together with the line $v u$. We say that a sequence is simple if it is either a path or a cycle; all other sequences are redundant. The length of a sequence is the number of occurrences of lines in it. An $n$-sequence is a sequence of length $n$. We take a point to be a 0 -path and a line to be a 1-path.

The line digraph $\&(D)$ of a digraph $D$ is a digraph whose points correspond to the lines of $D$ and whose lines are given by the rule: If $x_{i j}$ and $x_{m n}$ are lines of $D$, then a line is drawn in $\mathscr{L}(D)$ from (the point corresponding to) $x_{i j}$ to $x_{m n}$ if in $D$ the second point of $x_{i j}$ is the same as the first point of $x_{m n}$ (that is, $v_{j}=v_{m}$ ). Since $\mathscr{L}(D)$ is itself a digraph, we may also form its line digraph $\mathscr{L}(\mathscr{L}(D))$. We let $\mathscr{L}(\mathscr{L}(D))=\mathscr{L}^{2}(D)$, and in general $\mathcal{L}\left(\mathscr{L}^{n-1}(D)\right)=$ $\mathscr{L}^{n}(D)$. 
Let $L$ be a $k$-sequence of $D$. From the definition of $\mathscr{L}(D)$ we see that $L$ yields a $(k-1)$-sequence in $\mathscr{L}(D)$, a $(k-2)$-sequence in $\mathscr{L}^{2}(D)$, and in general, a $(k-n)$-sequence in $\mathcal{L}^{n}(D)$, for $n \leq k$. In particular, each $k$-sequence of $D$ yields a line in $\mathcal{L}^{k-1}(D)$ and a point in $\mathfrak{L}^{k}(D)$. These observations are illustrated by the digraphs shown in Fig. 1. The 2-path $L_{543}$ of $D$ yields in $\mathscr{L}(D)$ the line from $x_{54}$ to $x_{43}$ and in $\mathscr{L}^{2}(D)$ the point $y_{543}$. The 3 -cycle $L_{1521}$ of $D$ yields in $\mathscr{L}(D)$ the 2-path- $x_{15}, x_{52}, x_{21}$-and in $\mathscr{L}^{2}(D)$ the line from $y_{152}$ to $y_{521}$. And the redundant 3 -sequence $L_{5152}$ of $D$ yields in $\&(D)$ the 2-path- $x_{51}, x_{15}, x_{52}$-and in $\mathscr{L}^{2}(D)$ the line from $y_{515}$ to $y_{152}$.
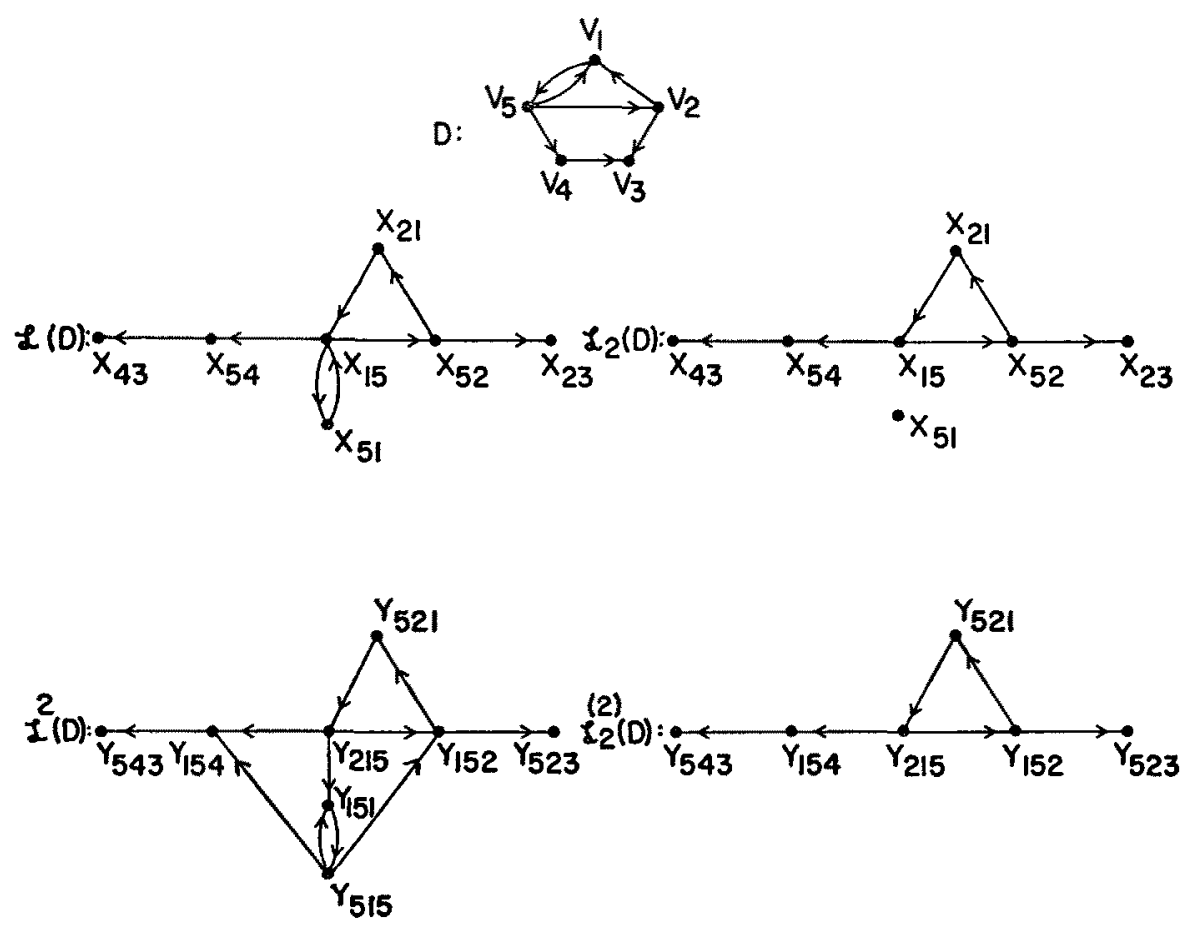

Figdre 1

Since there is a one-to-one correspondence between $k$-sequences of $D$ and lines in $\mathfrak{2}^{k-1}(D)$, it is possible to ascertain the number of $k$-sequences from ${\mathcal{L}^{k-1}}^{(D)}$. It is important to note that if $D$ has no cycles, then every $k$-sequence of $D$ is a $k$-path. In this case, the $k$-paths of $D$ correspond uniquely to the lines of $\mathfrak{L}^{k-1}(D)$. But if $D$ has any cycles of length less than $k$, some of the lines in $\mathscr{L}^{k-1}(D)$ will correspond to redundant sequences. Since we are here interested only in simple sequences, we want a method for obtaining a modified digraph, analogous to $\mathcal{2}^{k-1}(D)$, in which each line corresponds 
to a simple $k$-sequence of $D$, and we want to be able to distinguish between paths and cycles. We now describe such a method, leaving until later a detailed proof of its general applicability.

It is evident that every 2-sequence of a digraph is either a 2-path or a 2-cycle. Let $Z$ be a 2-cycle of $D$, as for example, the one in Figure 1 containing $v_{1}$ and $v_{5}$. Now there is a 2 -sequence beginning and ending at each of these points, each of which is a 2-cycle said to be rooted at the indicated point. The one, $L_{515}$, is rooted at $v_{5}$ and yields in $\mathcal{L}(D)$ the line from $x_{51}$ to $x_{15}$. The other, $L_{151}$, is rooted at $v_{1}$ and yields the line from $x_{15}$ to $x_{51}$. Note that these two lines form a 2-cycle in $\&(D)$. On the other hand, a 2-path in $D$ yields one line in $\mathscr{L}(D)$ that does not lie on a 2-cycle. We see, then, that each line in $\mathscr{L}(D)$ corresponds to a simple 2-sequence of $D$; it represents a 2-cycle of $D$ if it lies on a 2-cycle, and it represents a 2-path otherwise.

We want next to construct from $\mathscr{L}(D)$ a modified digraph, analogous to $\mathscr{L}^{2}(D)$, whose lines correspond only to simple 3 -sequences of $D$. Since a 3-sequence is simple if and only if it does not contain a 2-cycle, we may remove from $\mathscr{L}(D)$ all lines representing 2-cycles of $D$, namely, those lying on a 2-cycle (without, however, removing the first and second points of these lines). We denote the resulting digraph by $\mathfrak{L}_{2}(D)$. Clearly, every line in $\mathfrak{L}_{2}(D)$ uniquely corresponds to a 2-path. Let us now form the line digraph of $\mathscr{L}_{2}(D)$ and denote it by $\mathscr{L}_{2}^{(2)}(D)$. Each line in $\mathscr{L}_{2}^{(2)}(D)$ corresponds to two lines in $\mathfrak{L}_{2}(D)$ that have a point in common, which occurs if and only if the lines in $\mathfrak{L}_{2}(D)$ represent two 2-paths of $D$ having a line in common. Two such paths, taken together, form a 3-path if and only if the first point of one is different from the last point of the other. Otherwise, they form a 3-cycle. Thus, every line in $\mathfrak{L}_{2}^{(2)}(D)$ represents a simple 3-sequence of $D$.

We saw above that rooted 2-cycles of $D$ correspond to lines that lie on a 2 -cycle in $\&(D)$. We now show that, in a similar way, each rooted 3-cycle of $D$ yields a line in $\mathfrak{L}_{2}^{(2)}(D)$ that lies on a 3-cycle. Let $Z$ be a 3-cycle of $D$ containing the points $v_{i}, v_{j}$, and $v_{k}$. Clearly, there are three 2-paths of $D$ of the form: $L_{i j k}, L_{i k i}$, and $L_{k i j}$. Now, $L_{i j k}$ and $L_{i k i}$, taken together, form a 3-cycle rooted at $v_{i}$. They have the line $x_{i k}$ in common and yield in $\mathfrak{L}_{2}^{(2)}(D)$ a line from $y_{i j k}$ to $y_{i k i}$. Similarly, $L_{i k i}$ and $L_{k i j}$ form a 3-cycle rooted at $v_{i}$ and yield a line from $y_{i k i}$ to $y_{k i i}$. Finally, $L_{k i i}$ and $L_{i j k}$ form a 3-cycle rooted at $v_{k}$ and yield a line from $y_{k i}$; to $y_{i j k}$. It is clear, then, that each 3-cycle of $D$ yields three lines in $\mathscr{L}_{2}^{(2)}(D)$ which form a 3-cycle and each line of this 3 -cycle represents a rooted 3-cycle of $D$. Thus, the lines in $\mathscr{L}_{2}^{(2)}(D)$ provide the desired information concerning the simple 3-sequences of $D$; each line lying on a 3-cycle represents a rooted 3-cycle of $D$ and every other line corresponds to a 3-path.

These observations are illustrated in Fig. 1. Clearly, there is a one-to-one correspondence between the 2-paths of $D$ and the lines in $\mathscr{L}_{2}(D)$. There is, furthermore, a one-to-one correspondence between the simple 3-sequences 
of $D$ and the lines in $\mathscr{L}_{2}^{(2)}(D)$. Finally, we note that in $\mathscr{L}_{2}^{(2)}(D)$ each line not lying on a 3-cycle corresponds to a 3-path of $D$ whereas each line lying on a 3-cycle represents a rooted 3-cycle.

The procedure just described can easily be generalized. We take as the inductive hypothesis that there is a one-to-one correspondence between the simple $n$-sequences of $D$ and the lines of $\mathcal{L}_{n-1}^{(n-1)}(D)$. Let $\mathcal{R}_{n}^{(n-1)}(D)$ be the subgraph of $\mathcal{L}_{n-1}^{(n-1)}(D)$ obtained by removing the lines of all its $n$-cycles. Then each line in $\mathscr{L}_{n}^{(n-1)}(D)$ corresponds to an $n$-path of $D$. Let

$$
\mathscr{L}_{n}^{(n)}(D)=\mathscr{L}\left(\mathscr{L}_{n}^{(n-1)}(D)\right) .
$$

Now each line in $\mathscr{L}_{n}^{(n)}(D)$ corresponds to two $n$-paths of $D$ having $n-1$ lines in common. Clearly, these two paths together form a simple $(n+1)$ sequence of $D$. If the first point of one of these paths is the same as the last point of the other, they combine to form an $(n+1)$-cycle $Z$; and $Z$ contains $n+1$ rooted $(n+1)$-cycles, each of which yields a line lying on an $(n+1)$-cycle in $\mathcal{L}_{n}^{(n)}(D)$. Any line in $\mathcal{L}_{n}^{(n)}(D)$ not lying on an $(n+1)$-cycle must, therefore, correspond to an $(n+1)$-path of $D$.

We conclude, then, that the lines of $\mathfrak{L}_{k-1}^{(k-1)}(D)$ provide the desired information concerning simple $k$-sequences of $D$. That is, each line lying on a $k$-cycle uniquely corresponds to a rooted $k$-cycle of $D$, and every other line uniquely corresponds to a $k$-path of $D$. In the remainder of this paper we show how this information may be obtained by matrix methods.

\section{Matrix Operations}

Let $D$ be a digraph whose points are labeled $v_{1}, v_{2}, \cdots, v_{p}$. The adjacency matrix of $D, A=A(D)$, is a $p \times p$ matrix whose entries are $a_{i j}=1$ if there is a line $v_{i} v_{i}$ in $D$ and $a_{i i}=0$ otherwise. The matrix of simple $n$-sequences of $D, S_{n}=S_{n}(D)$, is also a $p \times p$ matrix whose entry $s_{i i}^{(n)}$ is the number of distinct $n$-paths from $v_{i}$ to $v_{i}$ when $i \neq j$, and whose entry $s_{i i}^{(n)}$ is the number of $n$-cycles rooted at $v_{i}$. Clearly, $S_{1}=A$. Given $A$, our problem is to find a series of matrix operations which will result in $S_{n}, 2 \leq n \leq p$.

In order to solve this problem we introduce the notion of the adjacency of two sequences of a digraph. If $L$ is a sequence of $D$, then the first point of $L$ will be denoted $\alpha(L)$, and the last point will be denoted $\omega(L)$. Let $L_{i}$ and $L_{j}$ be two sequences of $D$. We say that $L_{i}$ is $n$-adjacent to $L_{j}$ if there is an $n$-path $L_{e}$ which is a subpath of both $L_{i}$ and $L_{i}$ and which satisfies the conditions: $\omega\left(L_{e}\right)=\omega\left(L_{i}\right)$ and $\alpha\left(L_{e}\right)=\alpha\left(L_{i}\right)$. Now if $L_{i}$ is a simple $n$-sequence, then clearly $L_{i}=L_{0}$ and $L_{i}$ is a subpath of $L_{j}$ containing its first $n$ lines. Similarly, if $L_{i}$ is a simple $n$-sequence, then $L_{j}=L_{0}$ and $L_{i}$ is a subpath of $L_{i}$ consisting of its last $n$ lines. We saw above that there is a line from $L_{i}$ to $L_{i}$ in $\aleph_{n}^{(n)}(D)$ if and only if $L_{i}$ and $L_{i}$ are $n$-paths of $D$ having $n-1$ lines in common. In the present terminology, this means that each line from $L_{i}$ to $L_{j}$ in $\mathscr{L}_{n}^{(n)}(D)$ corresponds to two $n$-paths $L_{i}$ and $L_{i}$ of $D$ such that 
$L_{i}$ is $(n-1)$-adjacent to $L_{i}$. In the following discussion, we are concerned with the adjacency of the simple sequences of a digraph $D$.

Let $a$ be a square matrix whose rows and columns correspond to the simple $n$-sequences in $D$, for all $n=0,1, \cdots, p$. We assume an ordering $\theta$ of all simple sequences, and hence of the rows and columns of $a$, which is subject only to the restriction that every $n$-sequence precedes every $(n+1)$-sequence. By $\theta_{n}$ we shall mean $\theta$ restricted to the simple $n$-sequences. We denote by $A_{m n}$ the submatrix of $a$ whose rows correspond to simple $m$-sequences and whose columns correspond to simple $n$-sequences. Clearly, a can be expressed in terms of its submatrices as follows:

$$
\mathrm{Q}=\left[\begin{array}{cccccc}
A_{00} & A_{01} & \cdots & A_{0 n} & \cdots & A_{0_{p}} \\
A_{10} & A_{11} & \cdots & A_{1 n} & \cdots & A_{1 p} \\
\vdots & \vdots & & \vdots & & \vdots \\
A_{m 0} & A_{m 1} & \cdots & A_{m n} & \cdots & A_{m p} \\
\vdots & \vdots & & \vdots & & \vdots \\
A_{p 0} & A_{p 1} & \cdots & A_{p n} & \cdots & A_{p p}
\end{array}\right] .
$$

The entries $a_{i j}^{(m n)}$ of $A_{m n}$ are given by the rules

$$
a_{i j}^{(m n)}=1 \text { if }\left\{\begin{aligned}
& m<n \text { and } L_{i} \text { is } m \text {-adjacent to } L_{i}, \\
& m>n \text { and } L_{i} \text { is } n \text {-adjacent to } L_{i}, \\
& m=n, n>0, \text { and } L_{i} \text { and } L_{i} \text { are } n \text {-paths such that } \\
& L_{i} \text { is }(n-1) \text {-adjacent to } L_{i} \\
& m=n=0 \text { and the line } v_{i} v_{i} \text { is in } D ;
\end{aligned}\right.
$$

otherwise, $a_{i j}^{(m n)}=0$.

Note that for a given ordering $\theta$, the submatrix $A_{00}$ is the adjacency matrix of $D$, and the entire matrix $Q$ is well defined. We now show how the matrix $S_{n}$ can be obtained from certain submatrices of $a$, leaving until later the actual construction of $\alpha$ itself.

Consider the submatrix $A_{0 n}$ for $n>0$. Its $i$ th row corresponds to the point $v_{i}$ in $D$, its $k$ th column corresponds to the simple $n$-sequence $L_{k}$, and the entry $a_{i k}^{(0 n)}=1$ if and only if $v_{i}=\alpha\left(L_{k}\right)$. Likewise in the submatrix $A_{n 0}, a_{k i}^{(n 0)}=1$ if and only if $\omega\left(L_{k}\right)=v_{i}$. Thus, in the product $A_{0 n} A_{n 0}$ the term

$$
a_{i k}^{(0 n)} a_{k i}^{(n 0)}=1
$$

if and only if $L_{k}$ is a simple $n$-sequence from $v_{i}$ to $v_{i}$. If $v_{i}=v_{i}, L_{k}$ is an $n$-cycle rooted at $v_{i}$; otherwise $L_{k}$ is an $n$-path. Since the matrix $Q$ is defined so that all the simple $n$-sequences in $D$ are represented in the columns of $A_{0 n}$ 
and the rows of $A_{n 0}$, we see immediately that

$$
\sum_{k} a_{i k}^{(0 n)} a_{k j}^{(n 0)}=m
$$

if and only if there are $m$ simple $n$-sequences from $v_{i}$ to $v_{i}$ (i.e., $s_{i i}^{(n)}=m$ ). This essentially proves our first theorem.

THEOREM 1. For all $n>0$, the matrix of simple $n$-sequences is given by $S_{n}=A_{0 n} A_{n 0}$.

\begin{tabular}{|c|c|c|c|c|c|}
\hline$v_{1}$ & 000001 & 100000000 & 111000000 & 111000 & 0 \\
\hline & 10100 & 0110000 & 000100000 & 000110 & 1 \\
\hline & $A_{00}: 0000000$ & $A_{01}: 00000000$ & $A_{02}: 00000000000$ & $A_{03}: 00000000$ & $A_{04}: 0$ \\
\hline & 00100 & 0001000 & 00000000 & 000000 & 0 \\
\hline & 11010 & 00000111 & 00001111 & 000001 & 0 \\
\hline & 000001 & 0000111 & 111000000 & 1110000 & 0 \\
\hline & 10000 & 100000000 & 0000100000 & 000110 & 1 \\
\hline & 00100 & 00000000 & 000000000 & 0000000 & 0 \\
\hline & $\mathrm{A}_{10}: 001100$ & $A_{11}: 000000000$ & $A_{12}: 00000000000$ & $A_{13}: 00000000$ & $A_{14}: 0$ \\
\hline & 100000 & 10000000 & 00001000 & 0000000 & 0 \\
\hline & 01000 & 01100000 & 000000110 & 0000001 & 0 \\
\hline & 00010 & 000010000 & 00000001 & 0000000 & 0 \\
\hline$L_{15}$ & 100000 & 00000100 & $\begin{array}{llllllllll}0 & 0 & 0 & 0 & 0 & 0 & 0 & 0\end{array}$ & 00000000 & 0 \\
\hline $\mathrm{L}_{15}$ & 01000 & $\begin{array}{lllllllllll}0 & 0 & 0 & 0 & 0 & 1 & 0\end{array}$ & 00000110 & 110000 & 0 \\
\hline$=L_{154}$ & 00010 & 0000001 & 000000001 & 001000 & 0 \\
\hline $\mathrm{L}_{215}$ & 000001 & 10000000 & 011000000 & 000110 & 1 \\
\hline$L_{515}$ & $A_{20}: 000001$ & $A_{21}: 100000000$ & $A_{22}: 00000000000$ & $A_{23}: 00000000$ & $A_{24}: 0$ \\
\hline$I_{521}$ & 1000000 & 01000000 & 0000100000 & 00000001 & 0 \\
\hline $\mathrm{I}_{523}$ & 00100 & 0010000 & 00000000 & 000000 & 0 \\
\hline $\mathrm{L}_{543}$ & 00100 & 0001000 & 00000000 & 000000 & 0 \\
\hline $\mathrm{L}_{1521}$ & 100000 & 010000000 & 00000100 & 00000000 & 0 \\
\hline $\mathrm{L}_{1523}$ & 00100 & 00100000 & 000000010 & 00000000 & 0 \\
\hline$L_{1543}$ & 00100 & 00001000 & 00000001 & 00000000 & 0 \\
\hline $\mathrm{L}_{2152}$ & $A_{30}: 01000$ & $A_{31}: 0000010$ & $A_{32}: 01100000000$ & $A_{33}: 00000000$ & $A_{34}: 0$ \\
\hline $\mathrm{I}_{2154}$ & 000010 & 000000001 & 001000000 & 001000 & $\mathbf{1}$ \\
\hline $\mathrm{I}_{5215}$ & 000001 & 100000000 & 00001000000 & 00000000 & 0 \\
\hline$I_{21543}$ & $A_{40}: 000100$ & $A_{41}: 0001000$ & $A_{42}: 000000001$ & $A_{43}: 0010000$ & $A_{44}: 0$ \\
\hline
\end{tabular}

Figure 2 
Fig. 2 gives the matrix $\&$ for the digraph $D$ of Fig. 1. For convenience, each row is labeled so as to identify the corresponding simple sequence and, of course, the $k$ th row and the $k$ th column correspond to the same simple sequence. Thus, for example, the entry $a_{24}^{(02)}=1$ indicates that $v_{2}$ is the first point of $L_{215}$ and the entry $a_{45}^{(20)}=1$ means that $v_{5}$ is the last point of $L_{215}$.

Upon multiplying the appropriate submatrices of Fig. 2, we obtain the following matrices which give the number of $n$-paths and $n$-cycles, for each $n$, in the digraph of Fig. 1.

$$
\begin{aligned}
& A_{02} A_{20}=S_{2}=\left[\begin{array}{lllll}
1 & 1 & 0 & 1 & 0 \\
0 & 0 & 0 & 0 & 1 \\
0 & 0 & 0 & 0 & 0 \\
0 & 0 & 0 & 0 & 0 \\
1 & 0 & 2 & 0 & 1
\end{array}\right], \\
& A_{03} A_{30}=S_{3 \check{j}}=\left[\begin{array}{lllll}
1 & 0 & 2 & 0 & 0 \\
0 & 1 & 0 & 1 & 0 \\
0 & 0 & 0 & 0 & 0 \\
0 & 0 & 0 & 0 & 0 \\
0 & 0 & 0 & 0 & 1
\end{array}\right], \\
& A_{04} A_{40}=S_{4}=\left[\begin{array}{lllll}
0 & 0 & 0 & 0 & 0 \\
0 & 0 & 1 & 0 & 0 \\
0 & 0 & 0 & 0 & 0 \\
0 & 0 & 0 & 0 & 0 \\
0 & 0 & 0 & 0 & 0
\end{array}\right] .
\end{aligned}
$$

The only nonzero entries on the main diagonal of $S_{2}$ are $s_{11}^{(2)}=s_{55}^{(2)}=1$. This tells us that $D$ has only one 2-cycle and that it contains $v_{1}$ and $v_{s}$. The entry $s_{53}^{(2)}=2$ means that there are two 2-paths from $v_{5}$ to $v_{3}$, and inspection of $D$ shows these to be $L_{543}$ and $L_{523}$. In $S_{3}$ the entries on the main diagonal indicate that $D$ has one 3-cycle containing $v_{1}, v_{2}$, and $v_{5}$. The remaining nonzero entries give the number of 3-paths from $v_{i}$ to $v_{i}$. Finally, $S_{4}$ reveals that $D$ has only one 4-path and that it goes from $v_{2}$ to $v_{3}$.

We have seen that the matrix $S_{n}$ can be obtained from the submatrices $A_{0 n}$ and $A_{n 0}$ of $a$. Before describing how the entire matrix $Q$ can be constructed from the adjacency matrix $A_{00}$, we need to establish a number of structural relations among the submatrices of $Q$. 
We begin by considering $A_{n n}$ and $A_{n, n+1}$. The next theorem, concerning the adjacency of simple sequences in a digraph, shows that the unit entries of these two submatrices are related in a particular way.

THEOREM 2. In any digraph, the number of ordered pairs of n-paths such that the first is $(n-1)$-adjacent to the second is equal to the number of ordered pairs consisting of a simple n-sequence followed by a simple $(n+1)$ sequence such that the first is n-adjacent to the second.

Proof. Let $L_{i}$ and $L_{i}$ be any two $n$-paths such that $L_{i}$ is $(n-1)$ adjacent to $L_{j}$. Clearly, there is an $(n+1)$-sequence, $L_{v}$, which begins at $\alpha\left(L_{i}\right)$ and contains the line from $\alpha\left(L_{i}\right)$ to $\alpha\left(L_{i}\right)$ and the path $L_{i}$. Since all points of $L_{i}$ and of $L_{i}$ are distinct, $L_{\sigma}$ is simple. Moreover, there is just one $(n+1)$-sequence that contains both $L_{i}$ and $L_{i}$. Now since $\alpha\left(L_{i}\right)=\alpha\left(L_{o}\right)$ and $L_{a}$ contains $L_{i}$, it follows that $L_{i}$ is $n$-adjacent to $L_{a}$. Thus, for each ordered pair, $L_{i}$ and $L_{i}$, there is a unique ordered pair, $L_{i}$ and $L_{o}$, such that $L_{i}$ is $n$-adjacent to $L_{a}$.

Now assume that there exists an $n$-path $L_{i}$ which is $n$-adjacent to the simple $(n+1)$-sequence $L_{a}$. Let $L_{\hbar}$ be the first line of $L_{o}$ and let $L_{j}$ be the subsequence of $L_{v}$ from $\omega\left(L_{k}\right)$ to $\omega\left(L_{q}\right)$. Since the points of $L_{g}$, with the possible exception of $\alpha\left(L_{g}\right)$ and $\omega\left(L_{q}\right)$, are distinct, $L_{j}$ is an $n$-path. Moreover, $L_{i}$ and $L_{j}$ have a common $(n-1)$-path $L_{c}$. Since $\alpha\left(L_{e}\right)=\alpha\left(L_{i}\right)$ and $\omega\left(L_{e}\right)=\omega\left(L_{i}\right)$, it follows that $L_{i}$ is $(n-1)$-adjacent to $L_{i}$. There can be only one such pair, $L_{i}$ and $L_{i}$, since any simple $(n+1)$-sequence contains exactly one pair of $n$-paths.

Recall that (by definition) the entry $a_{i j}^{(n n)}=1$ indicates that the $n$-path $L_{i}$ is $(n-1)$-adjacent to the $n$-path $L_{i}$, and the entry $a_{i j}^{(n, n+1)}=1$ indicates that the simple $n$-sequence $L_{i}$ is $n$-adjacent to the simple $(n+1)$-sequence $L_{i}$. The following result thus follows immediately from Theorem 2 .

Conollary 2a. The number of unit entries in $A_{n n}$ equals the number of unit entries in $A_{n, n+1}$.

It is readily apparent that for each simple $(n+1)$-sequence in $D$ there is exactly one $n$-path that is $n$-adjacent to it. In other words, each column in $A_{n, n+1}$ contains exactly one unit entry, and we may conclude that the number of columns in $A_{n, n+1}$ equals the number of unit entries in $A_{n n}$. We see, then, that there is a one-to-one correspondence between the unit entries in $A_{n n}$ and the simple $(n+1)$-sequences of $D$. Hence any ordering, $\theta_{n+1}$, of one of these induces an ordering of the other.

Let us assume now that the $r \times r$ matrix $A_{n n}$ is known, for some $n$. By this assumption, there is an ordering $\theta_{n}$ of the simple $n$-sequences of $D$ and hence of the corresponding rows and columns of $a$. Let us arbitrarily order the unit entries of $A_{n n}$ by $\theta_{n+1}$. The submatrix $A_{n, n+1}$ can then be obtained from $A_{n n}$ in the following way.

Construction 1. If the $k$ th unit entry, relative to $\theta_{n+1}$, of $A_{n n}$ occurs 
in its $i$ th row, then $A_{n, n+1}$ is obtained by letting its $k$ th column be the $r \times 1$ vector which has 1 in its $i$ th place and 0 's elsewhere.

Clearly this construction satisfies the definition of $A_{n, n+1}$, for its rows and columns are ordered by $\theta_{n}$ and $\theta_{n+1}$ respectively and there is a 1 in the $i, k$ entry of the constructed matrix if and only if the $n$-path $L_{i}$ is $n$-adjacent to the simple $(n+1)$-sequence $L_{k}$.

The matrix $A_{n+1, n}$ can be constructed from $A_{n n}$ in a similar way. By an argument analogous to that in the proof of Theorem 2 it can be shown that the number of unit entries in $A_{n n}$ is the same as the number of unit entries in $A_{n+1, n}$ and there is exactly one unit entry in each row of $A_{n+1, n}$. We may, then, employ the same ordering $\theta_{n+1}$ as above and construct $A_{n+1, n}$ from $A_{n n}$.

Construction 2. If the $k$ th unit entry, relative to $\theta_{n+1}$, of $A_{n n}$ occurs in its $j$ th column, then $A_{n+1, n}$ is obtained by letting its $k$ th row be the $1 \times r$ vector which has 1 in its $j$ th place and 0 's elsewhere.

By this construction, there is a 1 in the $k, j$ entry of the obtained matrix if and only if the simple $(n+1)$-sequence $L_{k}$ is $n$-adjacent to the $n$-path $L_{i}$. Thus, the definition of $A_{n+1, n}$ is satisfied.

(The submatrices under consideration contain information relevant to our earlier discussion of line digraphs. The submatrix $A_{n n}$ contains the adjacency matrix of $\mathfrak{L}_{n}^{(n)}(D)$, as can be seen by comparing Figs. 1 and 2 . And if the transpose of a matrix $M$ is denoted $M^{\prime}$, then $A_{10}+A_{01}^{\prime}$ is obtained from the incidence matrix of $D$ by replacing every nonzero entry by +1 . Likewise, $A_{n+1, n}+A_{n, n+1}^{\prime}$ contains the incidence matrix of $\mathfrak{L}_{n}^{(n)}(D)$ as a submatrix.)

These two constructions may be illustrated by Fig. 2. The unit entry $a_{26}^{(22)}$ indicates that $L_{152}$ is 1 -adjacent to $L_{521}$. We let $\theta_{2}$ be the ordering of the unit entries of $A_{22}$ obtained in the following way. Start with the first row and read from left to right, noting the order of occurrence of unit entries; go to the second row and read from left to right, and continue to note the order of occurrence of unit entries; continue this procedure through the last row. We see that $a_{26}^{(22)}$ is the first unit entry. Thus, the first column of $A_{23}$ has a 1 in its second place and zeros elsewhere. Similarly, the first row of $A_{32}$ has a 1 in its sixth place and zeros elsewhere. The unit entry $a_{21}^{(23)}$ means that $L_{152}$ is 2-adjacent to a simple 3-sequence, namely, $L_{1521}$. And the entry $a_{16}^{(32)}$ means that $L_{1521}$ is 2-adjacent to a 2 -path, namely, $L_{521}$.

We next consider how the matrix $A_{n n}$ is related to $A_{n, n-1}$ and $A_{n-1, n}$. By definition, the rows and columns of $A_{n n}$ correspond to the simple $n$-sequences in $D$, and $a_{i j}^{(n n)}=1$ if and only if $L_{i}$ and $L_{i}$ are $n$-paths and $L_{i}$ is $(n-1)$-adjacent to $L_{j}$. In the product $A_{n, n-1} A_{n-1, n}$, the rows and columns also correspond to the simple $n$-sequences in $D$, and each $i, j$ entry is given by

$$
\sum_{0} a_{i e}^{(n, n-1)} a_{e i}^{(n-1, n)} .
$$


Now, $a_{i \varepsilon}^{(n, n-1)} a_{e j}^{(n-1, n)}=1$ if and only if $L_{\varepsilon}$ is an $(n-1)$-path such that $L_{i}$ is $(n-1)$-adjacent to $L_{s}$ and $L_{0}$ is $(n-1)$-adjacent to $L_{j}$, or equivalently $L_{i}$ is $(n-1)$-adjacent to $L_{i}$. Since for any pair, $L_{i}$ and $L_{i}$, there can be only one $L_{0}$ which satisfies the above conditions, every entry in $A_{n, n-1} A_{n-1, n}$ is either 0 or 1 . Thus the only difference between $A_{n, n-1} A_{n-1, n}$ and $A_{n n}$ is that unit entries in the former represent the $(n-1)$-adjacency of two simple $n$-sequences whereas in the latter they represent the $(n-1)$-adjacency of two $n$-paths. Hence if in $A_{n, n-1}$ and $A_{n-1, n}$ we change to 0 all the unit entries involving $n$-cycles and denote the resulting matrices by $B_{n, n-1}$ and $B_{n-1, n}$, then $A_{n n}$ is obtained by forming the product $B_{n, n-1} B_{n-1, n}$. These observations constitute a proof of the next theorem. (Since the unit entries in $B_{n, n-1}$ and $B_{n-1, n}$ represent only $n$-paths, use of these matrices has the effect of removing $n$-cycles from $\mathfrak{L}_{n-1}^{(n-1)}(D)$.)

TheOREM 3. For all $n \geq 1$, the matrix $A_{n n}$ is the product

$$
A_{n n}=B_{n, n-1} B_{n-1, n+}
$$

The next theorem shows that $B_{n, n-1}$ and $B_{n-1, n}$ can be obtained by matrix operations which identify the unit entries involving $n$-cycles in $A_{n, n-1}$ and $A_{n-1, n}$. We rely on two matrix operations not previously used: (a) the transpose of $M$, denoted $M^{\prime}$, and (b) the element-wise product $M \times N$, where both $M$ and $N$ have $r$ rows and $c$ columns and the $i, j$ entry of $M \times N$ is given by $m_{i j} \cdot n_{i i}$.

Since our definition of a digraph precludes 1-cycles, it is immediate that $A_{10}=B_{10}$ and $A_{01}=B_{01}$.

THEorem 4. For all $n \geq 2$, the matrices $B_{n, n-1}$ and $B_{n-1, n}$ are given by the equations

$$
B_{n, n-1}=A_{n, n-1}-\left[A_{n, n-1} \times\left(A_{n-1,0} A_{0 n}\right)^{\prime}\right],
$$

and

$$
B_{n-1, n}=A_{n-1, n}-\left[A_{n-1, n} \times\left(A_{n 0} A_{0, n-1}\right)^{\prime}\right] .
$$

Proof. Consider first the matrix $\left(A_{n-1,0} A_{0 n}\right)^{\prime}$, whose entries are given by

$$
\left(\sum_{e} a_{i e}^{(n-1,0)} a_{e i}^{(0, n)}\right)^{\prime} .
$$

Now, $a_{i s}^{(n-1,0)}=1$ if and only if the simple $(n-1)$-sequence $L_{i}$ is 0 -adjacent to the 0 -sequence $L_{e}$, that is $\omega\left(L_{j}\right)=L_{t}$. And $a_{s i}^{(0, n)}=1$ if and only if $L_{c}$ is 0-adjacent to the simple $n$-sequence $L_{i}$, that is, $\alpha\left(L_{i}\right)=L_{\text {o }}$. Clearly, there can be at most one point $L_{\mathrm{e}}$ satisfying both these conditions. Hence, every entry in $\left(A_{n-1,0} A_{\mathfrak{o n}_{n}}\right)^{\prime}$ is either 0 or 1 , and each unit entry, $i, j$, indicates that $\alpha\left(L_{i}\right)=\omega\left(L_{i}\right)$. By definition, $a_{i i}^{(n, n-1)}=1$ if and only if $L_{i}$ is $(n-1)$-adjacent to $L_{i}$, that is, $\omega\left(L_{i}\right)=\omega\left(L_{i}\right)$. Since the transpose 
of $\left(A_{n-1,0} A_{0 n}\right)$ has the same number of rows and columns as $A_{n-1, n}$, we may form the element-wise product of these matrices to obtain the matrix given by the bracketed term in the first equation of the theorem. Now, the $i, j$ entry of this matrix is 1 if and only if $\alpha\left(L_{i}\right)=\omega\left(L_{i}\right)$ and $\omega\left(L_{i}\right)=\omega\left(L_{i}\right)$ or, equivalently, $\alpha\left(L_{i}\right)=\omega\left(L_{i}\right)$. Since $L_{i}$ is a simple $n$-sequence, it follows that $L_{i}$ is an $n$-cycle. Thus, each $i, j$ unit entry in this matrix corresponds to an $n$-cycle $L_{i}$ that is $(n-1)$-adjacent to an $(n-1)$-sequence $L_{i}$. Upon subtracting this matrix from $A_{n, n-1}$, we obtain a matrix satisfying the definition of $B_{n, n-1}$.

A similar argument establishes the second equation of the theorem.

Up to this point we have considered how the submatrices $A_{n_{n}}$, which lie on the main diagonal of $Q$, are related to submatrices immediately adjacent to the main diagonal. We now show that any submatrix $A_{m n}, m \neq n$, is related in a particular way to certain other submatrices of $a$. The next two lemmas concerning the adjacency of sequences in a digraph provide a basis for establishing these relationships.

Lemma 1. Let $L_{0}, L_{1}, \cdots, L_{r}$ be a series of sequences such that $L_{0}$ is an $(m+e)$-sequence, for all $e=0,1, \cdots, r$. If $L_{e}$ is $(m+e)$-adjacent to $L_{e+1}$, for all $e=0,1, \cdots, r-1$, then $L_{0}$ is m-adjacent to $L_{r}$.

Proof. If $L_{0}$ is m-adjacent to $L_{1}$ and $L_{1}$ is $(m+1)$-adjacent to $L_{2}$, then $L_{1}$ must be an $(m+1)$-path containing $L_{0}$. But since $L_{1}$ is contained in $L_{2}$ and $\alpha\left(L_{0}\right)=\alpha\left(L_{1}\right)=\alpha\left(L_{2}\right)$, we see that $L_{0}$ is $m$-adjacent to $L_{2}$. Now, if $L_{0}$ is $m$-adjacent to $L_{r-1}$ and $L_{r-1}$ is $(m+r-1)$-adjacent to $L_{r}$, then $L_{r-1}$ must be an $(m+r-1)$-path contained in $L$. Since

$$
\alpha\left(L_{0}\right)=\alpha\left(L_{r-1}\right)=\alpha\left(L_{r}\right),
$$

it follows that $L_{0}$ is $m$-adjacent to $L_{r}$.

The next lemma is in the nature of a converse to Lemma 1.

LEMMA 2. Let $L_{0}$ be an m-path and $L_{r}$ a simple $n$-sequence. If $L_{0}$ is $m$-adjacent to $L_{r}$, then there exists a unique series of sequences $L_{0}, L_{1}, \cdots, L_{r}$ such that $L_{a}$ is $(m+e)$-adjacent to $L_{s+1}$, for all $e=0,1, \cdots, r-1$.

Proof. For any $e=0,1, \cdots, r$ let $L_{e+1}$ be the $(m+e)$-subpath of $L_{r}$ for which $\alpha\left(L_{e}\right)=\alpha\left(L_{r}\right)$. Since $L_{c}$ is contained in $L_{t+1}$ and $\alpha\left(L_{e}\right)=\alpha\left(L_{e+1}\right)$, $L_{0}$ is $(m+e)$-adjacent to $L_{e+1}$, Thus the paths $L_{e}$ form a series $L_{0}, L_{1}, \cdots$, $L_{r}$. That this series is unique follows from the fact that $L_{0}$ is the only $(m+e)$-subpath of $L_{r}$ for which $\alpha\left(L_{e}\right)=\alpha\left(L_{r}\right)$.

Theorem 5. For any $m<n$,

$$
\begin{aligned}
& A_{m n}=A_{m, m+1} A_{m+1, m+2} \cdots A_{n+1, n}, \\
& A_{n m}=A_{n, n-1} A_{n-1, n-2} \cdots A_{m+1, m} .
\end{aligned}
$$

Proor. The entries of the matrix $A_{m, m+1} A_{m+1, m+2} \cdots A_{n-1, n}$ are composed of products of the form $a_{i f}^{(m, m+1)} a_{f a}^{(m+1, m+2)} \cdots a_{h i}^{(n-1, n)}$. Clearly 
this product must be 0 or 1 . If it equals 1 , then there is an $n$-sequence $L_{i}$ in $D$ such that $L_{i}$ is $m$-adjacent to the $(m+1)$-sequence $L_{f}$. Likewise, $L_{f}$ is $(m+1)$-adjacent to the $(m+2)$-sequence in $L_{o}$. This series continues until we come to the $(n-1)$-sequence $L_{k}$ which is $(m+n-1)$-adjacent to the simple $n$-sequence $L_{i}$. By Lemma $1, L_{i}$ is $m$-adjacent to $L_{i}$. By Lemma 2 , the series described in the above product is unique. Thus the $i, j$ entry of $A_{m, m+1} A_{m+1, m+2} \cdots A_{n+1, n}$ is either 0 or 1 , and if it is 1 , then $a_{i i}^{(m n)}=1$. If $a_{i i}^{(m n)}=1$, then $L_{i}$ is $m$-adjacent to $L_{i}$. By Lemma 2 we may construct a series $L_{i}, L_{1}, L_{2}, \cdots, L_{e}, \cdots, L_{i}$ such that $L_{e}$ is $(m+e)$-adjacent to $L_{e+1}$. Thus $a_{i 1}^{(m, m+1)} a_{12}^{(m+1, m+2)} \cdots a_{r i}^{(n-1, n)}=1$, and since this series is unique the $i, j$ entry of $A_{m, m+1} A_{m+1, m+2} \cdots A_{n-1, n}$ is 1 .

This establishes the validity of statement (1) of the theorem. A similar argument holds for statement (2).

It will be recalled that Theorem 1 shows that the matrix $S_{n}$ can be obtained from the submatrices $A_{0 n}$ and $A_{n 0}$. The following corollary of Theorem 5 therefore provides information useful in finding $S_{n}$.

Corollarry 5a. For any $n=2,3, \cdots, p$,

$$
\begin{aligned}
& A_{0 n}=A_{0, n-1} A_{n-1, n}, \\
& A_{n 0}=A_{n, n-1} A_{n-1,0} .
\end{aligned}
$$

Proof. By Theorem 5,

$$
A_{0 n}=A_{01} A_{12} \cdots A_{n-2, n-1} A_{n-1, n},
$$

But

$$
A_{01} A_{12} \cdots A_{n-2, n-1}=A_{0, n-1} .
$$

This establishes (1), and a similar argument establishes (2).

We summarize the above material and the constructions involved by the following algorithm.

Algorithm 1. Let $D$ be a digraph and $\theta_{n}$ be an arbitrary ordering of the simple $n$-sequences of $D$. Then the matrix $S_{n}$ of simple $n$-sequences of $D$, for $n>0$, can be obtained by the following procedure.

1. Order the points of $D$ by $\theta_{0}$ and construct $A_{00}$.

2. Order the unit entries of $A_{00}$ by $\theta_{1}$ and construct $A_{01}$ and $A_{10}$.

3. Construct $A_{11}$ by the formula

$$
A_{11}=A_{10} A_{01} \text {. }
$$

Steps 4-7 give a recursive procedure for finding $A_{n n}, n=2,3, \cdots, p$.

4. Order the unit entries of $A_{n-1, n-1}$ by $\theta_{n}$ and construct $A_{n-1, n}$ and $A_{n, n-1}$.

5. Construct $A_{0 n}$ and $A_{n 0}$ by the formulas

$$
\begin{aligned}
& A_{0 n}=A_{0, n-1} A_{n-1, n}, \\
& A_{n 0}=A_{n, n-1} A_{n-1,0} .
\end{aligned}
$$


6. Construct $B_{n-1, n}$ and $B_{n, n-1}$ by the formulas

$$
\begin{aligned}
& B_{n-1, n}=A_{n-1, n}-\left[A_{n-1, n} \times\left(A_{n 0} A_{0, n-1}\right)^{\prime}\right], \\
& B_{n, n-1}=A_{n, n-1}-\left[A_{n, n-1} \times\left(A_{n-1,0} A_{0 n}\right)^{\prime}\right] .
\end{aligned}
$$

7. Construct $A_{n n}$ by the formula

$$
A_{n n}=B_{n, n-1} B_{n-1, n} .
$$

8. If $A_{n n}=[0]$ or $n=p$, terminate the procedure. Otherwise, return to step 4.

9. Construct $S_{n}$ from the results of step 5 by the formula

$$
S_{n}=A_{0 n} A_{n 0}
$$

Clearly, Step 1 of this algorithm is simply the customary way of constructing the adjacency matrix of a digraph. Steps 2 and 4 are justified by Corollary 2a, together with Constructions 1 and 2. Steps 3 and 7 make use of Theorem 3. Step 5 is justified by Corollary 5a. Step 6 results from Theorem 4. And, finally, Step 9 is given by Theorem 1 .

As noted above, the matrix $a$ of Figure 2 is obtained from the digraph of Fig. 1. In constructing $Q$, we have used a standard procedure for the ordering $\theta_{n+1}$ of the unit entries of $A_{n n}$, for all $n>0$, as follows. Start with the first row and read from left to right, noting the order of occurrences of unit entries; go to the second row and read from left to right and continue to note the order of occurrence of unit entries; continue similarly through the last row. For completeness, we have presented all submatrices of $a$, but not all of these are required for finding the matrix $S_{n}$ of simple $n$-sequences of $D$. In fact, the algorithm yields only submatrices of $Q$ lying on the first row (that is, $\left.A_{\text {on }}\right)$, the first column $\left(A_{n 0}\right)$, the main diagonal $\left(A_{n n}\right)$, and the diagonals adjacent to the main diagonal $\left(A_{n, n+1}\right.$ and $\left.A_{n+1, n}\right)$.

In using the algorithm to obtain $S_{n}$ for even moderately large digraphs, Step 6 involves considerable calculation. Our final theorem shows that under certain conditions this step may be eliminated.

Theorem 6. If the number of nonzero rows in $A_{0 n}$ is less than $n$, then

$$
A_{n n}=A_{n, n-1} A_{n-1, n} \text {. }
$$

Proof. The entries of $A_{0 n}$ indicate which points in $D$ are the first points of the simple $n$-sequences corresponding to the columns of $A_{0 m}$. If there is a $k$-cycle in $D$ for $k \geq n$, then there must be at least $n$ points which are the first points of simple $n$-sequences. Thus if the number of nonzero rows is less than $n$, there can be no $k$-cycles in $D$ for all $k \geq n$. Hence, $B_{n, n-1}=A_{n, n-1}$ and $B_{n-1, n}=A_{n-1, n}$, and the equation of the theorem follows from Theorem 3 . 


\section{Some Related Matrices}

Once the matrix $S_{n}$ of simple $n$-sequences of a digraph is obtained for each $n$, it is relatively easy to construct other useful matrices. We now briefly discuss some of these.

The matrix of simple sequences of a digraph $D, S(D)$, is a $p \times p$ matrix whose entry $s_{i j}$ is the number of distinct paths (of any length) in $D$ from $v_{i}$ to $v_{j}$ when $i \neq j$, and whose entry $s_{i i}$ is the number of cycles containing $v_{i}$. Clearly, then, we have

$$
\sum_{n=1}^{p-1} S_{n}=S(D)
$$

The distance from $v_{i}$ to $v_{j}$, denoted $d_{i i}$, is the length of a shortest path from $v_{i}$ to $v_{i}$. If there is no path from $v_{i}$ to $v_{i}$, we let $d_{i j}=\infty$. The distance matrix of $D$, denoted $N(D)$, is a $p \times p$ matrix whose entries are the distances $d_{i j}$. The following statements are readily established.

1. Every diagonal entry $d_{i i}$ of $N(D)$ is 0 .

2. $d_{i j}=\infty$ if there is no entry $s_{i j}^{(n)} \neq 0$ in $S_{n}$, for $n \leq p-1$.

3. Otherwise, $d_{i i}$ is the smallest value of $n$ such that $s_{i j}^{(n)} \neq 0$.

To construct the distance matrix $N(D)$ for the digraph of Fig. 1, we begin by entering 0 's on the main diagonal. Next, we transfer the unit entries in $A_{00}$ to $N(D)$. Then we enter 2's in all empty locations of $N(D)$ whenever there is a nonzero entry in $S_{2}$. The next step is to enter 3's in all empty locations whenever there is a nonzero entry in $S_{3}$. Since each nondiagonal entry in $S_{4}$ is 0 , we complete $N(D)$ by entering $\infty$ in the remaining empty locations. The resulting matrix is

$$
N(D)=\left[\begin{array}{ccccc}
0 & 2 & 3 & 2 & 1 \\
1 & 0 & 1 & 3 & 2 \\
\infty & \infty & 0 & \infty & \infty \\
\infty & \infty & 1 & 0 & \infty \\
1 & 1 & 2 & 1 & 0
\end{array}\right] .
$$

A detour from $v_{i}$ to $v_{i}$ is a path of maximum length from $v_{i}$ to $v_{i}$. The detour matrix of $D$ is a $p \times p$ matrix $E(D)=\left[e_{i}\right]$, where $e_{i i}=\infty$ if there is no path from $v_{i}$ to $v_{i}$ and otherwise $e_{i j}$ is the length of a detour from $v_{i}$ to $v_{i}$. To construct $E(D)$ we use the following immediate observations.

1. Every diagonal entry $e_{i i}$ is 0 .

2. $e_{i j}=\infty$ if there is no entry $s_{i j}^{(n)} \neq 0$ in $S_{n}$, for $n \leq p-1$.

3. Otherwise, $e_{i j}$ is the maximum integer $n$ such that $s_{i i}^{(n)} \neq 0$. 


\section{A Generalization of the Method}

An (irreflexive) network $N$ is a digraph together with an assignment of some value to each of its lines. Such a value, for example, may be a probability, an integer, or a positive or negative sign. Thus, the value of a line may represent the probability of going directly from one state to another, the cast of traveling directly from one place to another, or the signed quality of affection of one person for another. (For a discussion of various interpretations of networks, see Harary, Norman, and Cartwright ([2], ch. 12 and 13).) Given a network with a particular value system for its lines, the question arises as to how to assign values to its sequences.

If, for example, the values assigned to the lines of a network are probabilities, it is customary to define the value of a sequence from $v_{i}$ to $v_{i}$ as the product of the values of its lines, which if the values are independent is the probability of going from $v_{i}$ to $v_{i}$ via this sequence. And if there are several sequences from $v_{i}$ to $v_{i}$, we add the values of these sequences to obtain the probability of going from $v_{i}$ to $v_{i}$ by any of them.

Or, if the values of a network represent costs, the value of a sequence would be obtained by summing the values of its lines to give the cost of traveling along the entire sequence. Now, if there were several sequences from $v_{i}$ to $v_{i}$ and one wanted to know the minimum cost of traveling from $v_{i}$ to $v_{i}$, one would find the minimum value of all these sequences.

In this section, we present a generalization of Theorem 1 which, for a variety of value systems, allows one to determine some function of the values of all simple $n$-sequences in a network.

We begin by presenting a generalized arithmetic, whose specific meaning will depend upon the value system of the network in question. For any network there must be a rule of combination whereby the lines of a sequence are used to form the value of a sequence. We call this generalized multiplication and denote it by $\odot$. Thus, if $t$ and $t^{\prime}$ are the values on the lines of a 2-sequence, $t \odot t^{\prime}$ would be the value of the sequence itself. Likewise, if there are several sequences between two points, there must be some way of assigning a value which represents the set of all such sequences. Such an assignment is called generalized addition and is denoted by $\oplus$. Thus, if $t$ and $t^{\prime}$ are the values of two sequences from $v_{i}$ to $v_{j}$, their generalized sum is $t \oplus t^{\prime}$.

We assume that these operations satisfy the following conditions. First, they are associative and commutative. Second, there is an identity element 1 , with respect to generalized multiplication, which follows the rule

$$
t \odot \underline{1}=t .
$$

Third, there is an identity element $\ddot{0}$, with respect to generalized addition, 
which follows the rule

$$
t \oplus \ddot{0}=t
$$

Finally, we have the condition

$$
t \odot \ddot{0}=\ddot{0} .
$$

In using this arithmetic, we shall make use of the generalized product of two matrices $A \odot B$. By $A \odot B$ we shall mean the matrix whose $i, j$ entry is given by the expression

$$
\left(a_{i 1} \odot b_{1 i}\right) \oplus\left(a_{i 2} \odot b_{2 j}\right) \oplus \cdots \oplus\left(a_{i r} \odot b_{r i}\right)=\ddot{\sum}_{k=1}^{k=r} a_{i k} \odot b_{k i} .
$$

The value matrix of a network $N$ is the matrix, $M(N)=M$, whose entry $m_{i j}$ is the value of the line from $v_{i}$ to $v_{i}$ if such a line is in $N$ and 0 otherwise. We illustrate the use of our generalized arithmetic for two rather different value systems. Consider first a network whose values are costs. In this case, the entry $m_{i j}$ of the value matrix is the cost of traveling directly from $v_{i}$ to $v_{i}$ if this is possible. If this is not possible, $m_{i i}=\infty$. Suppose that we want to find the minimum cost of traveling from $v_{i}$ to $v_{i}$ in $n$ steps. Using a procedure introduced by Hasse [3], we use the operation of ordinary addition for generalized multiplication, and we use the procedure of taking the minimum value of a set of values for generalized addition. Thus, in this specification of our generalized arithmetic, the identity element 0 is $\infty$, and 1 is 0 . Now if we form the product $M \odot M=M^{\text {(21 }}$, we get

$$
\begin{aligned}
m_{i j}^{[2]} & =\left(m_{i 1} \odot m_{1 j}\right) \oplus\left(m_{i 2} \odot m_{2 j}\right) \oplus \cdots \oplus\left(m_{i p} \odot m_{p i}\right) \\
& =\min \left(m_{i 1}+m_{1 j}, \quad m_{i 2}+m_{2 i}, \cdots, m_{i p}+m_{p i}\right),
\end{aligned}
$$

which gives the minimum cost of traveling from $v_{i}$ to $v_{i}$ in 2 steps, and in general $M^{[n]}$ gives the minimum costs for $n$ steps. As a second example, consider a network whose values are probabilities. Now we use ordinary multiplication for $\odot$, ordinary addition for $\oplus$, and we use 0 and 1 for the identity elements $\ddot{0}$ and $\ddot{1}$, respectively. With these operations $m_{i i}^{[n]}$ gives the probability of going from $v_{i}$ to $v_{i}$ by any sequence of length $n$. It is clear that various specifications of our generalized arithmetic may be employed, depending upon the nature of the value system of a network.

We continue to develop our generalization by introducing three new matrices. First, we have the value matrix of simple $n$-sequences $M\left(S_{n}\right)$ whose $i, j$ entries are the generalized sums of the values of all the simple $n$-sequences from $v_{i}$ to $v_{i}$ in $N$. Second $F_{n}, 1 \leq n \leq p$, is a square matrix whose diagonal entries $f_{i i}^{(n)}$ are the values of the simple $n$-sequences $L_{i}$, relative to the ordering $\theta_{n}$, and whose off-diagonal entries $f_{i j}^{(n)}$ are 0 . Finally, $G_{n}, 1 \leq n \leq p$, is a square matrix whose diagonal entries $g_{i i}^{(n)}$ are the values of the last line of the simple $n$-sequences $L_{1}$, relative to the ordering $\theta_{n}$, and whose entries $g_{i i}^{(n)}$ are $\ddot{0}$. 
If we are given the value matrix $M$ for a network $N$, we can readily obtain the adjacency matrix $A(N)$ by changing every $\ddot{0}$ to 0 and letting all other values equal 1 . We can then construct the matrix $a$ for the underlying digraph of the network $N$. In the following discussion, we need to modify a as follows: Let $\ddot{A}_{m n}$ be the matrix obtained from $A_{m n}$ by replacing each occurrence of 0 by 0 and each occurrence of 1 by $\ddot{1}$. The importance of this modification will soon be apparent.

Now suppose that $F_{n}$ and $G_{n}$ are known and let

$$
H_{n}=\ddot{A}_{n n} \odot G_{n} \text {. }
$$

By the definition of $\odot$ we have

$$
h_{i j}^{(n)}=\sum_{k} \ddot{a}_{i k}^{(n n)} \odot g_{k i}^{(n)} .
$$

But by the definition of $G_{n}$ there is only one value of $k$ such that $g_{k i}^{(n)} \neq \ddot{0}$. Hence

$$
h_{i j}^{(n)}=\ddot{a}_{i j}^{(n n)} \odot g_{j i}^{(n)} .
$$

If $\ddot{a}_{i j}^{(n n)}=\ddot{1}$, then $h_{i j}^{(n)}=g_{j i}^{(n)}$. Consequently the last line of the $(n+1)$ sequence formed by the $n$-paths $L_{i}$ and $L_{j}$ has the value $g_{i}$. Clearly if $\ddot{a}_{i i}^{(n n)}=0$, then $L_{i}$ is not $(n-1)$-adjacent to $L_{i}$, and the entry $h_{i i}^{(n)}=\ddot{0}$, reflects this fact.

Now let

$$
T_{n n}=F_{n} \odot H_{n} .
$$

Again applying the definition of $\odot$ we have

$$
t_{i i}^{(n n)}=\sum_{i} f_{i k}^{(n)} \odot h_{k i}^{(n)} .
$$

By definition of $F_{n}$ there is only one value of $k$ such that $f_{i k}^{(n)} \neq \ddot{0}$. Hence by (9) and (7) we get,

$$
\begin{aligned}
t_{i j}^{(n n)} & =f_{i i}^{(n)} \odot h_{i i}^{(n)} \\
& =f_{i i}^{(n)} \odot \ddot{a}_{i j}^{(n)} \odot g_{i j}^{(n)} .
\end{aligned}
$$

We have seen before that $g_{i j}^{(n)}$ is the value of the last line of the $n$-path $L_{i}$ and hence the value of the last line of the simple $(n+1)$-sequence formed by $L_{i}$ and $L_{i}$. Likewise, $f_{i i}^{(n)}$ is the value of the $n$-path of $L_{i}$ and hence the value of the first $n$ lines of the larger sequence. Thus the value of the simple $(n+1)$-sequence corresponding to $\ddot{a}_{i j}^{(n n)}=\ddot{1}$ is the product

$$
\begin{aligned}
f_{i i}^{(n)} \odot \vec{a}_{i i}^{(n)} \odot g_{i j}^{(n)} & =f_{i i}^{(n)} \odot \ddot{1} \odot g_{i i}^{(n)} \\
& =f_{i i}^{(n)} \odot g_{j i}^{(n)} .
\end{aligned}
$$

Consequently we see that $T_{n n}$ is a kind of generalization of $A_{n n}$. 
These results give us a means of constructing the matrices $F_{n+1}$ and $G_{n+1}$ from $T_{n n}$ and $H_{n}$. Assume that $F_{n}, G_{n}$, and $A_{n n}$ are known. From these matrices we can calculate $T_{n n}$ and $H_{n}$ according to (5) and (8). Once these matrices are obtained, we then order their non-0 entries by $\theta_{n+1}$. Then $F_{n+1}$ may be constructed from $T_{n n}$ in the following way.

Construction 3. If the kth non-0 entry of $T_{n n}$, relative to $\theta_{n+1}$, is $t$, then $F_{n+1}$ is obtained by letting its $k$ th row have the value $t$ in its $k$ th place and 0 elsewhere.

The matrix $G_{n+1}$ may be constructed from $H_{n}$ by a similar procedure, as follows.

Construction 4. If the kth non-0̈ entry of $H_{n}$, relative to $\theta_{n+1}$, is $t$, then $G_{n+1}$ is obtained by letting its $k$ th row have the value $t$ in its $k$ th place and 0 elsewhere.

We are now able to state our generalization of Theorem 1.

Theorem 7 . For any $n \geq 2$, the value matrix of simple $n$-sequences is given by

$$
M\left(S_{n}\right)=\ddot{A}_{0 n} \odot F_{n} \odot \ddot{A}_{n 0} .
$$

Proof. By definition, the $i, j$ entry of $\ddot{A}_{0 n} \odot F_{n}$ is

$$
\sum_{k} \tilde{a}_{i k}^{(0 n)} \odot f_{k i}^{(n)} .
$$

Since $f_{k i}^{(n)}=\ddot{0}$ for all $k \neq j$, the above expression becomes simply

$$
\ddot{a}_{i j}^{(0))} \odot f_{i i}^{(n)} .
$$

Thus, $\ddot{A}_{0 n} \odot F_{n} \odot \ddot{A}_{n 0}$ has as its $i, j$ entry

$$
\sum_{k} \ddot{a}_{i k}^{(0 n)} \odot f_{k k}^{(n)} \odot \ddot{a}_{k i}^{(n 0)},
$$

which may be rewritten

$$
\ddot{\sum}_{k} f_{k k}^{(n)} \odot \ddot{a}_{i k}^{(0 n)} \odot \ddot{a}_{k i}^{(n 0)} .
$$

Clearly,

$$
\ddot{a}_{i k}^{(0 n)} \odot \ddot{a}_{k j}^{(n 0)}=\ddot{1}
$$

if and only if there is a simple $n$-sequence from $v_{i}$ to $v_{i}$ in $N$ (Theorem 1). Hence, the $i, j$ entry of

$$
\ddot{A}_{0 n} \odot F_{n} \odot \ddot{A}_{n 0}
$$

is the generalized sum of the values of the simple $n$-sequences from $v_{i}$ to $v_{i}$ in $N$.

In conclusion, we present an algorithm for finding the value matrix 
of simple $n$-sequences of a network. This algorithm is stated in terms of our generalized arithmetic. In specific applications one must, of course, choose appropriate interpretations for the operations of $\odot$ and $\oplus$ and for the identity elements $\ddot{0}$ and $\ddot{1}$. This algorithm makes use of certain steps of Algorithm 1 and employs both binary matrices and value matrices.

Algorithm 2. Let $N$ be a network and $\theta_{n}$ be an arbitrary ordering of the simple $n$-sequences of $N$. Then the value matrix $M\left(S_{n}\right)$ of simple $n$-sequences of $N$, for $n>0$, can be obtained by the following procedure.

1. Order the points of $N$ by $\theta_{0}$ and construct $A_{00}$ and $M$.

2. Use step 2 of Algorithm 1.

2a. Order the non-0 entries of $M$ by $\theta_{1}$ and construct $F_{1}$ using Construction 3. Note that $F_{1}=G_{1}$.

3. Use step 3 of Algorithm 1.

Steps 3a-7 give a recursive procedure for finding $A_{n n}, n=2,3, \cdots, p$.

3a. Convert $A_{n-1, n-1}$ to $\ddot{A}_{n-1, n-1}$.

3b. Construct $H_{n-1}$ by the formula

$$
H_{n-1}=\ddot{A}_{n-1, n-1} \odot G_{n-1} .
$$

3e. Construct $T_{n-1, n-1}$ by the formula

$$
T_{n-1, n-1}=F_{n-1} \odot H_{n-1} .
$$

4. Use step 4 of Algorithm 1.

4a. Order the non-0 entries of $T_{n-1, n-1}$ by $\theta_{n}$ and construct $F_{n}$ using Construction 3.

4b. Order the non- $\ddot{0}$ entries of $H_{n-1}$ by $\theta_{n}$ and construct $G_{n}$ using Construction 4.

5. Use step 5 of Algorithm 1.

5a. Convert $A_{0 n}$ and $A_{n 0}$ to $\ddot{A}_{0 n}$ and $\ddot{A}_{n 0}$.

6. Use step 6 of Algorithm 1.

7. Use step 7 of Algorithm 1.

8. If $A_{n \mathrm{a}}=[0]$ or $n=p$, terminate the procedure. Otherwise, return to step 3a.

9. Construct $M\left(S_{n}\right)$ from the results of steps $5 \mathrm{a}$ and $4 \mathrm{a}$ by the formula

$$
M\left(S_{n}\right)=\ddot{A}_{0 n} \odot F_{n} \odot \ddot{A}_{n o} .
$$

\section{Discussion}

The algorithms presented here are useful in obtaining information about a variety of structural properties that are based on nonredundant sequences. The matrix $S_{n}$ of simple $n$-sequences of $D$, which is obtained by Algorithm 1, gives the number of $n$-paths from $v_{i}$ to $v_{i}$ and the number of $n$-cycles containing $v_{i}$. Of special interest are the matrices $S_{p-1}$ and $S_{p}$, where $p$ is the number of points in $D$, for one can ascertain from these matrices 
respectively the number of complete paths and the number of complete cycles in $D$. From the matrices $S_{n}$, for $n \leq p$, one can construct the matrix $S$ of simple sequences which gives the number of paths and cycles of any length in $D$, the distance matrix which gives the length of a shortest path from $v_{i}$ to $v_{i}$, and the detour matrix which gives the length of a longest path from $v_{i}$ to $v_{i}$. One can also ascertain the length of a shortest cycle and of a longest cycle in $D$.

Algorithm 2 is applicable when values are associated with the lines of a digraph. If these values are probabilities, the matrix $M\left(S_{n}\right)$, when obtained by use of the appropriate arithmetic, gives the probability of going from $v_{i}$ to $v_{i}$ along a path of length $n$. And if $n$ is the distance from $v_{i}$ to $v_{i}$, the $i, j$ entry is the probability of going from $v_{i}$ to $v_{i}$ in the smallest possible number of steps. When the values of lines are costs, one can obtain a matrix $M\left(S_{n}\right)$ whose $i, j$ entry gives the cost of a cheapest simple sequence of length $n$ from $v_{i}$ to $v_{i}$. From the matrices $M\left(S_{n}\right)$, for $n \leq p$, it is possible to ascertain the least cost of any path from $v_{i}$ to $v_{i}$ and of any cycle containing $v_{i}$. With suitable modifications, similar information can be obtained concerning paths and cycles of maximal cost. Finally, we note that Algorithm 2 can be used when positive or negative signs are assigned to lines. In this case, the $i, j$ entry of $M\left(S_{n}\right)$ indicates the number of positive and of negative paths of length $n$ from $v_{i}$ to $v_{i}$. Such information is useful in the study of the path balance of a structure as developed by Harary, Norman, and Cartwright [2].

The major practical limitation of these algorithms lies in the fact that the rows and columns of the generated matrices correspond to simple sequences of $D$. Hence, if $D$ has a great many simple sequences of given length, the resulting matrix is large. We have prepared computer programs which ean quickly process digraphs containing up to 5,000 simple sequences of a given length. However, the task remains to develop procedures for larger numbers of simple sequences.

\section{REFERENCES}

[1] Harary, F. and Norman, R. Z. Some properties of line digraphs. Rendiconti del Circolo Mathematico di Palermo, 1960, 9, 161-168.

[2] Harary, F., Norman, R. Z., and Cartwright, D. Structural models: An introduction to the theory of directed graphs. New York: Wiley, 1965.

[3] Hasse, Maria. Über die Behandlung graphentheorischer Probleme unter Verwendung der Matrizenrechnung. Wiss. Z. Techn. Univer. Dresden, 1961, 10, 1313-1316.

[4] Luce, R. D. and Perry, A. D. A method of matrix analysis of group structure. Psychometrika, 1949, 14, 95-116.

[5] Parthasarathy, K. R. Enumeration of paths in digraphs. Psychometrika, 1964, 29, 153-165.

[6] Ross, I. C. and Harary, F. On the determination of redundancies in sociometric chains. Psychometrika, 1952, 17, 195-208. 